\title{
Lumbar Plexus
}

National Cancer Institute

\section{Source}

National Cancer Institute. Lumbar Plexus. NCI Thesaurus. Code C12845.

A network of nerves lying within the substance of the psoas muscle that is formed by the ventral rami of L1-L4, and which provides motor and sensory contributions to the anterior and medial compartments of the leg, abdominal wall, and pelvic areas. 